\title{
Adhesion force Imaging in air and liquid by adhesion mode atomic force microscopy
}

\author{
Kees O. van der Werf, Constant A. J. Putman, Bart G. de Grooth, and Jan Greve \\ Department of Applied Physics, University of Twente, P. O. Box 217, 7500 AE Enschede, The Netherlands
}

(Received 17 May 1994; accepted for publication 6 July 1994)

\begin{abstract}
A new imaging mode for the atomic force microscope (AFM), yielding images mapping the adhesion force between tip and sample, is introduced. The adhesion mode AFM takes a force curve at each pixel by ramping a piezoactuator, moving the silicon-nitride tip up and down towards the sample. During the retrace the tip leaves the sample with an adhesion dip showing up in the force curve. Adhesion force images mapping parameters describing this adhesion dip, such as peak value, width, and area, are acquired on-line together with the sample topography. Imaging in air gives information on the differences in hydrophobicity of sample features. While imaging a mercaptopentadecane-gold layer on glass in demineralized water, the adhesion force could be modulated by adding phosphate buffered saline.
\end{abstract}

Atomic force microscopy (AFM) is well known for its high-resolution topographic images of a wide variety of samples. To acquire these topographic images several modes of operation have been developed over the past 8 years. These include contact mode $\mathrm{AFM},{ }^{1}$ noncontact mode $\mathrm{AFM},{ }^{2}$ and tapping mode AFM in air ${ }^{3}$ and liquid. ${ }^{4,5}$ The information accessible to AFM-type systems, however, is not only limited to topography. Electrostatic, ${ }^{6}$ magnetic, ${ }^{7}$ and friction forces $^{8}$ can also be mapped with submicron resolution. Moreover, it has been shown that by introducing a force modulation between tip and sample in contact mode AFM, it is possible to obtain information on the viscoelastic properties of samples. ${ }^{9-11}$

One of the problems any AFM operator encounters is the large adhesion force due to the formation of a water meniscus between tip and sample in ambient conditions (both are covered by a thin water film). This force can be quantified by registrating force curves; $;^{12,13}$ values up to $100 \mathrm{nN}$ are common. The magnitude strongly depends on the contact area between sample and tip, type of sample surface, and the relative humidity. Upon immersing the tip and sample in liquid the adhesion forces are reduced to the $\mathrm{nN}$ level. For imaging of soft samples with low loading forces using contact mode AFM, the remaining forces can be further reduced by changing the $\mathrm{pH}$, the ion concentration or ion type. ${ }^{14,15}$ The very nature of these adhesion forces, however, may provide valuable information on the tip-sample interaction which is sensitive to the chemical composition of both tip and sample surface.

In this letter we introduce the mapping of adhesion forces between tip and sample as a new AFM imaging mode. Basically, force curves are determined at each pixel in the image. From the force curves several parameters describing the adhesion between tip and sample are extracted. Topographic and adhesion force images are acquired simultaneously on-line in the adhesion mode AFM.

In a modified version of our stand-alone $\mathrm{AFM}^{16}$ the silicon-nitride tip (radius of curvature $20-50 \mathrm{~nm}$ ) is moved in and out of contact, by ramping a small piezoactuator (connected to the main piezotube), which is placed in the cantilever holder. The tip is lowered towards the sample. Upon touching the sample the cantilever deflects until a preset cantilever deflection setpoint is reached (see Fig. 1). The piezotravel up to this setpoint is monitored and is a measure of the sample topography $(h)$; on top of a bump the tip has to be lowered less far to reach the setpoint, then above a hole. After the setpoint has been reached the tip is retracted to the upper limit allowed by the ramping voltage. During this retrace the tip leaves the sample at some point, resulting in the well-known adhesion dip. From the retrace curves the following parameters describing the adhesion forces are obtained: peak value of the adhesion dip $p$, width of this $\operatorname{dip} w$, and area of the adhesion dip $a$ (see Fig. 1). All these functions were implementing in electronic hardware. A pixel clock triggers a ramp generator coupled to the piezo actuator.

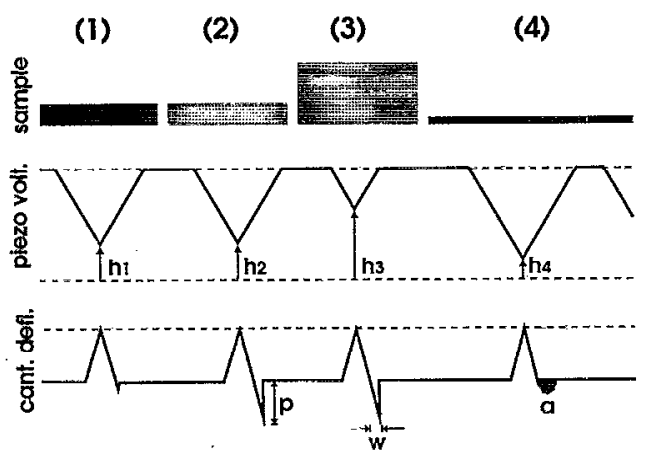

FIG. 1. Schematic illustration of the operation of the adhesion mode atomic force microscope. Variations in height $(h)$ and in chemical composition of the sample surface (top) are detected. (1) The tip is lowered towards and onto the sample up to the force setpoint (bottom). Then the tip moves up by reversing the ramping voltage on the piezo (middle). During the upward movement the tip leaves the surface, with only a little adhesion. (2) Compared to (1) there is no change in height, so the applied piezovoltage is the same (middle). The adhesion force, however, is much larger (bottom). In situation (3) the adhesion stays the same, but the ramping voltage is smaller due to the increase in height. Finally, the lower part of the sample (middle; high applied ramping voltage) is covered by an adsorbate, e.g., a layer of proteins such as poly-L-lysine, giving a typical adhesion dip as sketched (4). The adhesion dip is described by the peak $p$, the width $w$, and the area $a$. 

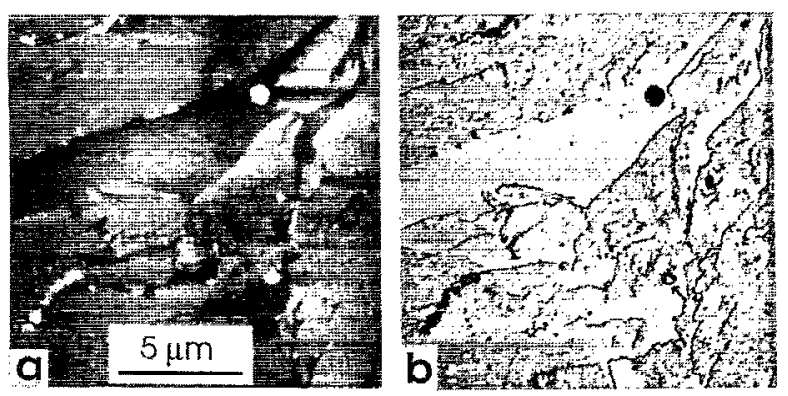

FIG. 2. Height (a) and adhesion force (b) images of a Langmuir-Blodgett monolayer film of diethylene glycol diamine pentacosadinoic amide (DPDA), polymerized by UV irradiation, on a microscope glass slide as obtained by adhesion mode AFM. The adhesion force image depicts the peak value of the adhesion dip. The force constant of the $100-\mu \mathrm{m}$-long cantilever was $0.6 \mathrm{~N} / \mathrm{m}$, the acquisition rate was 550 force curves per $\mathrm{s}$ and the image size is $300 \times 300$ datapoints. The grey scale corresponds to $15 \mathrm{~nm}$ in (a) and $50 \mathrm{nN}$ in (b). Arrow: see text. The fine raster superimposed in (a) is due to electronic airfacts of the high-voltage amplifier.

Upon reaching the sample deflection setpoint, the voltage applied to the piezo is stored in a sample/hold circuit and the ramping voltage reversed. The peak value of the adhesion dip $p$ during the retrace curve is measured by a peak detector. The width of the dip $w$ is determined by an integrator coupled to a comparator, which is started and stopped when the deflection signal crosses a discriminator signal level set just below the "zero"-deflection level. During the same period a second integrator integrates the deflection signal yielding the area of the adhesion dip $a$.

Figure 2 shows topographic and adhesion force images of a Langmuir-Blodgett monolayer film of diethylene glycol diamine pentacosadinoic amide (DPDA), polymerized by ultraviolet (UV) irradiation, ${ }^{17}$ on a microscope glass slide. Force curves were recorded at a rate of 550 per s. The thickness of the film is about $5 \mathrm{~nm}$ [Fig. 2(a)]. The adhesion force image [Fig. 2(b)] does not show the absolute adhesion force, because a background level is subtracted and the remaining differences are magnified. The average adhesion force on the glass is $300 \mathrm{nN}$ (the relative humidity was about $75 \%$ ) and the force variations in Fig. 2(b) are $30 \mathrm{nN}$. We clearly observe that the adhesion force between tip and DPDA (270 $\mathrm{nN}$ ) is less than the adhesion force between tip and glass. So DPDA is lcss hydrophylic than glass. This was to be expected, because the hydrophylic tail groups of the amphiphilic DPDA molecules are directed towards the glass. This example illustrates that an AFM operating in this adhesion force mode in air is capable of discriminating between objects on the basis of differences in adhesion forces resulting from the lack or presence of a water film. We do observe also some changes in adhesion forces which are not related to differences in hydrophobicity. The adhesion force is also influcnced by changes in contact arca resulting in a decreased adhesion force at step edges and on objects. On top of these objects the contact area increases and so does the adhesion force (arrow in Fig. 2).

Whereas imaging while in air the adhesion forces are predominantly due to differences in hydrophobicity, in liquid it is possible to directly measure the tip-sample interaction due to Van der Waals forces and specific chemical interac-

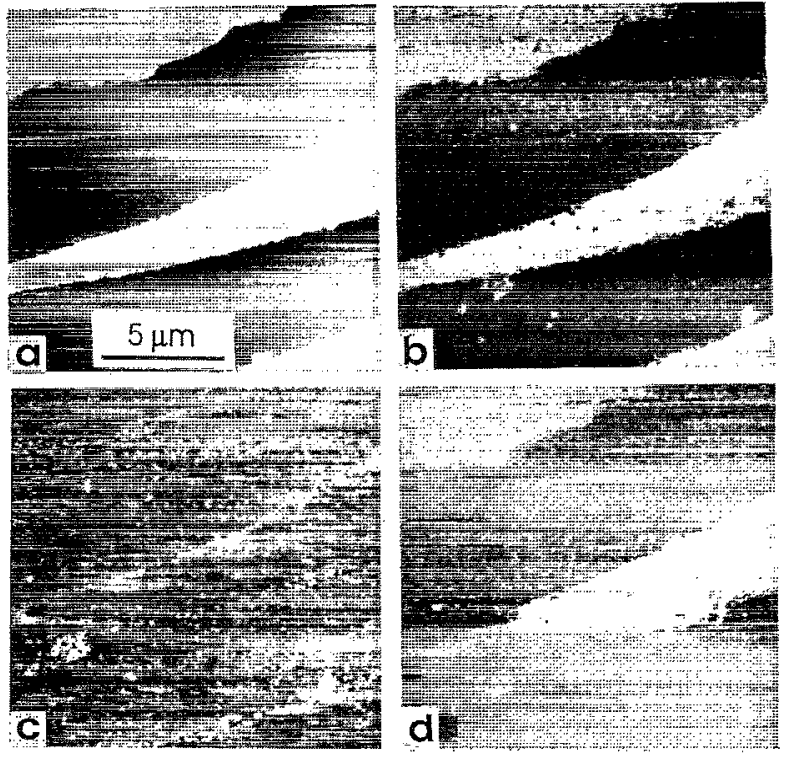

FIG. 3. Height (a) and adhesion force (b,c,d) images of a mercaptopentadecane-gold layer on glass in liquid obtained by adhesion mode AFM. The adhesion force images depict the peak values of the adlresion dips. (a,b) Were imaged in demineralized water. The thickness of the mercaptopentadecane-gold film is $45 \mathrm{~nm}$ and the difference in adhesion between glass and film is on the average $1-2 \mathrm{nN}$, with peaks up to $5 \mathrm{nN}$. (c) Adhesion image on the same area obtained in PBS. No adhesion on the mercaptopentadecane-gold film is detected anymore. (d) While imaging in demiwater (adhesion forces on mercaptopentadecane range from 0.5 to 1 $\mathrm{nN}$ ) some PBS was added and the adhesion force contrast disappears. The force constant of the $200-\mu \mathrm{m}$-long cantilever was $0.06 \mathrm{~N} / \mathrm{m}$, the acquisition rate was 70 force curves per $s$ and the image sizes are $300 \times 300$ datapoints.

tions. As a test sample we coated a glass slide with a thin gold layer. On top of the gold layer a self-assembled monolayer was formed by immersing the sample overnight in a solution of $1 \mathrm{mM} 15$-mercaptopentadecane in ethanol. Some parts of this mercaptopentadecane-gold layer were mechanically removed. In Figs. 3(a) and 3(b) the topographic and adhesion force images obtained simultaneously are shown. Force curves were recorded at a rate of 70 per $\mathrm{s}$. This rate is limited by the viscous drag of the cantilever in the water. Figures 3(a) and 3(b) are obtained in demineralized water. A clear difference in adhesion force for the mercaptopentadecane and the uncovered glass can be observed. The average adhesion force on the mercaptopentadecane layer is $1-2 \mathrm{nN}$, with some adhesion peaks reaching values up to $5 \mathrm{nN}$ [right side of Fig. 3(b)]. When changing from demiwater to phosphate buffered saline (PBS; $\mathrm{pH} 7.35$ ) the contrast in the adhesion force image disappeared completely [Fig. 3(c)]. Only some small features, such as the two cluster-like structure at the bottom (both are not visible in the height image), remain visible. This is in agreement with observations by Hoh et al. who showed that adhesion forces diminish by adding ions. ${ }^{13}$ The same area was imaged again in demiwater and we observed that the contrast in the adhesion force image was restored (data not shown). A striking image illustrating this effect is shown in Fig. 3(d). The upper half has been imaged in demiwater. The average adhesion forces are now between 
0.5 and $1 \mathrm{nN}$. This is lower than in Fig. 3(b) which is probably due to some ions still being present in the medium or to contamination of the tip. Halfway during the scan we added about $200 \mu \mathrm{l}$ of PBS to the sample cup (liquid volume of 1.5 $\mathrm{ml}$ ). As a result the adhesion force decreases below the detection limit [lower half of Fig. 3(d)].

The shape of the force curves mapped in the adhesion images of Figs. 2 and 3 were all similar to (2) and (3) in Fig. 1. Since the adhesion dip is triangular shaped the images mapping the peak, width, and area of the adhesion dip all look similar. In other experiments, however, we did encounter retrace curves as sketched in Fig. 1 (situation -4-). Adhesion dips of this nature, e.g., caused by the presence on the sample of absorbate molecules, such as poly-L-lysine- can be traced when closely comparing the different adhesion images (peak, width, and area). A more direct way of tracing such. adhesion dips is by determining the ratio between the width and peak height. Adhesion dips such as in situation (4) (Fig. 1) with deviations from the triangular shape will directly show up in such an image.

For two reasons it is essential that there is a force control that limits the maximum force on the sample (directly determined by the preset cantilever deflection setpoint) instead of a free-running oscillator ramping the tip up and down. If a free-running oscillator is used topographic changes dircetly result in changes in applied force. First, high loading forces on soft (biological) samples tend to be disruptive. Second, we sometimes observed that the adhesion force increased upon increasing the loading force. On soft deformable samples it may be assumed that a higher loading force leads to a larger contact area between tip and sample, and as a direct consequence to higher adhesion forces. The same effect has been observed on air-dried samples and the mercaptopentadecane film. Since the contact area will no longer increase (at the level of loading forces used), this effect has to be related to the increase in contact time. So it seems to be important to have a force control, which keeps the force as well a the contact time constant. A more or less similar force control has been used previously to image contact holes on integrated circuit devices with a hopping mode operation of the AFM. ${ }^{18}$

At a ramping rate of $70 \mathrm{~Hz}$ the force sensitivity of our current setup (in liquid) is limited to about $0.1 \mathrm{nN}$ (force constant of the cantilever is $0.06 \mathrm{~N} / \mathrm{m}$; Digital Instruments Inc., Santa Barbara, Ca). This limit is determined by mechanical resonances exciting the cantilever originating from ramping the piezo. Moreover, the lateral scanning movement of the cantilever in the liquid also causes vertical cantilever motions. Although the current force sensitivity is not suffi- cient to detect the breaking of $\mathrm{H}$ bonds (about $1.2 \times 10^{-11} \mathrm{~N}$ as measured by $A F M)^{19}$ while imaging, it is adequate to detect the rupture force of a single streptavidin-biotin interaction $(0.3 \mathrm{nN}) .^{20}$ In our future experiments with the adhesion mode AFM in liquid the main emphasis will be on molecular recognition interactions at the molecular level. We envision the imaging of cells with a biochemically functionalized tip, ${ }^{20}$ enabling a direct recognition of molecules, e.g., antigens, on the basis of the measured adhesion force. ${ }^{21}$

We thank Dave van de Heuvel for the preparation of the gold sample. The Langmuir-Blodgett sample was a kind gift of Hermann Gaub. This work has been supported in part by the Dutch Organization for Scientific Research NWO (C.A.J.P.).

${ }^{\text {l}}$ G. Binnig, C. F. Quate, and Ch. Gerber, Phys. Rev. Lett. 56, 930 (1986).

${ }^{2}$ Y. Martin, C. C. Williams, and H. K. Wickramasinghe, J. Appl. Phys. 61, 4723 (1987).

${ }^{3}$ Q. Zhong, D. Inniss, K. Kjoller, and V. B. Elings, Surf. Sci. Lett. 290 , L688 (1993).

${ }^{4}$ C. A. J. Putman, K. O. Van der Werf, B. G. De Grooth, N. F. Van Hulst, and J. Greve, Appl. Phys. Lett. 64, 2454 (1994).

${ }^{5}$ P. K. Hansma, J. P. Cleveland, M. Radmacher, D. A. Walters, P. Hillner, M. Beaznilla, M. Fritz, D. Vie, H. G. Hansma, C. B. Prater, J. Massie, L. Fukunaga, J. Gurley, and V. B. Elings, Appl. Phys. Lett. 64, 1738 (1994).

${ }^{6}$ F. Saurenbach and B. D. Terris, Appl. Phys. Lett. 56, 1703 (1990).

${ }^{7}$ P. Grütter, D. Rugar, and H. J. Mamin, Ultramicroscopy 47, 393 (1992).

${ }^{8}$ R. M. Overney, E. Meyer, J. Frommer, D. Brodbeck, R. Lüthi, L. Howald, H.-J. Güntherrodt, M. Fujihira, H. Takano, and Y. Gotoh, Nature 359, 133 (1992).

${ }^{9}$ P. Maivald, H.-J. Butt, S.A.C. Gould, C. B. Prater, B. Drake, J. A. Gurley, V. B. Elings, and P. K. Hansma, Nanotechnology 2, 103 (1991).

${ }^{10}$ M. Radmacher, R. W. Tillmann, and H. E. Gaub, Biophys. J. 64, 735 (1993).

${ }^{11}$ E.-L. Florin, M, Radmacher, B. Fleck, and H. E. Gaub, Rev. Sci. Instrum. 65, 639 (1994).

${ }^{12}$ A. L. Weisenhorn, P. K. Hansma, T. R. Albrecht, and C. F. Quate, Appl. Phys. Lett. 54, 2651 (1989).

${ }^{13}$ A. L. Weisenhorn, P. Maivald, H.-J. Butt, and P. K. Hansma, Phys. Rev. B. 45, 11226 (1992)

${ }^{14}$ J. H. Hoh, J.-P. Revel, and P. K. Hansma, Nanotechnology 2, 119 (1991).

${ }^{15}$ H.-J. Butt, Biophys. J. 60, 1438 (1991).

${ }^{1.6}$ K. O. Van der Werf, C. A. J. Putman, B. G. De Grooth, F. B. Segerink, E. H. Schipper, N. F. Van Hulst, and J. Greve, Rev. Sci. Instrum. 64, 2892 (1993).

${ }^{17}$ R. W. Tillmann, M. Radmacher, H. E. Gaub, P. Kenney, and H. O. Ribi, J. Phys. Chem. 97, 2928 (1993).

${ }^{18}$ H. Kado, S. Yamamoto, K. Yokoyama, and T. Tohda, J. Appl. Phys. 74, 4354 (1993).

${ }^{19}$ J. H. Hoh, J. P. Cleveland, C. B. Prater, J.-P. Revel, and P. K. Hansma, J. Amer. Chem. Soc. 114, 4917 (1992).

${ }^{20}$ G. U. Lee, D. A. Kidwell, and R. J. Colton, Langmuir 10, 354 (1994).

${ }^{21}$ During the preparation of this letter we received a preprint of a paper which describes a somewhat similar new mode of force imaging. In that approach each force curve is sampled at 100 points and the resulting data set is used to calculate off-line the various adhesion force images; Radmacher et al., Biophys. J. (in press). 